\title{
THE ROLE OF ANXIOLYTICS IN THE TREATMENT OF PATIENTS WITH ARTERIAL HYPERTENSION ACCOMPANIED BY ANXIETY DISORDERS
}

\author{
D. Komsiyska ${ }^{2}$, M. Penev ${ }^{1}$, D. Petrov ${ }^{3}$, M. Hristova ${ }^{1 *}$ \\ ${ }^{1}$ Department of Internal Medicine, Medical Faculty, Trakia University, Stara Zagora, Bulgaria \\ ${ }^{2}$ Department of Psychiatry and Neurology, Medical Faculty, Trakia University, Stara Zagora, Bulgaria \\ ${ }^{3}$ Department of Social Medicine and Health Management, Medical Faculty, Trakia University, Stara \\ Zagora, Bulgaria
}

\begin{abstract}
Previous studies have suggested a possible association between chronic exposure to stress and increased blood pressure. The mechanisms are incompletely understood. There are two main stress responses in the human body: the acute stress response (sympathetic-adrenal medullary pathway) and the chronic stress response (hypothalamo-pituitary-adrenal pathway).

The purpose of this study was to investigate the effect of chronic stress on blood pressure control as well as the benefits of adding anxiolytics to the standard antihypertensive therapy.

The study includes 202 patients who visited a psychiatrist's office regarding anxiety symptoms accompanied by high blood pressure occurring especially at night. The difference between the age for women $\left(\overline{\mathrm{x}}_{f}=66,34\right)$ and men $\left(\overline{\mathrm{x}}_{m}=60,14\right)$ is statistically significant $(\mathrm{t}(200)=-2.830, \mathrm{p}=$ $0.006<0.05) ; 20.3 \%$ had close relatives with psychiatric disorders. $94.6 \%$ of the patients had longstanding hypertension; $78.22 \%$ were regularly taking antihypertensive drugs. The presence of anxiety was confirmed by Spielberger's State-Trait Anxiety Inventory (STAI). The patients were treated with four types of anxiolytic drugs with different modes of action, prescribed individually or in combination. The results indicate that regardless of the choice of anxiolytic agent, the blood pressure of over $90 \%$ of the patients was stabilized.

The results suggest that anxiolytics, in addition to antihypertensive drugs, can produce clinically significant reductions in $\mathrm{BP}$ and improve emotional health in hypertensive patients with anxiety symptoms.
\end{abstract}

Key words: anxiolytics, arterial hypertension, chronic stress, anxiety symptoms

\section{INTRODUCTION}

Studies have suggested that chronic exposure to stress may have an influence on increased blood pressure (1) and favor the occurrence of cardiovascular disease; mechanisms are incompletely understood (2). Lucini et al. believe that symptoms related to chronic psychosocial stress would are related to autonomic dysregulation. The combination of sympathetic predominance, vagal withdrawal, and blunted baroreflex sensitivity might represent a link between psychosocial factors and future incidence of hypertension (2). Scattered throughout the literature are reports indicating that acute and chronic stress can activate renin and increase plasma levels of

\footnotetext{
*Correspondence to: Mariyana Kostova Hristova, 2 Ivan Kirov Vazov Street Apt.14, Zheleznik Quarter, 6014 Stara Zagora, Bulgaria, Phone:
} 0896 329990, Email address: MC_girl.87@abv.bg components of the renin-angiotensin system (RAS) (3). Groeschel M. and Braam B. propose that recurrent acute stress and chronic stress can induce inflammation due to angiotensin II-mediated activation of inflammatory cells, specifically monocytes and lymphocytes (3). Harrison et al. report that several studies have strengthened the concept that hypertension has an immunological basis (4). Paul J. Marvar et al. (2012) believe that T lymphocytes are essential for development of hypertension and that the central nervous system contributes to peripheral $\mathrm{T}$ lymphocyte activation and vascular inflammation (5). Daisy L Daubert et al. believe that catecholaminergic neurons within the central nervous system (located in the hindbrain area) are an integral part of stress-related neurocircuitry, and the nucleus of the solitary tract (NTS) plays a critical role in 
cardiovascular regulation. These authors have shown that lesioning NTS catecholaminergic neurons increases baseline blood pressure and causes an exaggerated blood pressure response to acute or repeated psychological stress, suggesting that physiological or pathophysiological inhibition of these neurons could lead to exaggerated stress responses and hypertension (6). Bremmer MA et al. believe that there is an association between major depression and blood cortisol levels. According to them, in younger adults (less than 65 years old), depression has been associated with hypercortisolemia. In older depressed patients, however, both low and high cortisol levels have been reported. These authors examined the possibility of a U-shaped association between depression and cortisol in older people, suggesting both hypo- and hyperactivity of the hypothalamic-pituitaryadrenal axis $(7,8)$. On the other hand, Manenschijn L et al. have correlated the cortisol levels in the scalp hair of 283 elderly participants to their cardiovascular disease history, and propose that long-term elevated cortisol may be an important cardiovascular risk factor (9). The Whitehall II prospective cohort study suggests that the risk of hypertension increases with repeated experience of depressive episodes over time and becomes evident in later adulthood (10). The CARDIA study has shown that young adults who show a large blood pressure (BP) response to psychological stress may be at risk for developing essential hypertension as they approach (11).

\section{MATERIALS AND METHODS}

The purpose of this study was to investigate chronic psycho-emotional stress as a possible etiological factor in the etiopathogenesis of essential hypertension and the effect of stress reduction by medication on blood pressure control.

The study group consisted of 202 patients with affective symptoms and an anamnesis of experiencing high nervous tension caused by some negative physical or emotional impact who visited a psychiatrist's office during the years 2012-2014. The patients reported inadequate blood pressure control despite taking antihypertensive therapy.

We categorized blood pressure for this purpose as well controlled (systolic blood pressure of $<140 \mathrm{~mm} \mathrm{Hg}$ and diastolic blood pressure of $<90 \mathrm{~mm} \mathrm{Hg}$ ) or poorly controlled (systolic blood pressure of $\geq 140 \mathrm{~mm} \mathrm{Hg}$ or diastolic blood pressure of $\geq 90 \mathrm{~mm} \mathrm{Hg}$ ).

Cognitive-behavioral therapy was not used in addition to the anxiolytic therapy in our study. Evaluation of symptoms of depression and anxiety

Psychiatric disorders were diagnosed by a psychiatrist (Table 1) using different methods based on observation and asking questions. The diagnoses were established according to the criteria of the International Statistical Classification of Diseases and Related Health Problems (ICD) The presence of anxiety was confirmed by Spielberger's State-Trait Anxiety Inventory (STAI) (Table 2).

Table 1. Diagnosis of mental disorders

\begin{tabular}{|ll|l|l|l|l|}
\hline & Frequency & Percent & Valid Percent & $\begin{array}{l}\text { Cumulative } \\
\text { Percent }\end{array}$ \\
\hline Valid & $\begin{array}{l}\text { Recurrent depressive } \\
\text { disorder }\end{array}$ & 156 & 77.2 & 77.2 & 77.2 \\
& $\begin{array}{l}\text { Panic disorder } \\
\text { Anxiety-depressive } \\
\text { disorders }\end{array}$ & 10 & 5.0 & 5.0 & 82.2 \\
& $\begin{array}{l}\text { Generalized anxiety } \\
\text { disorder }\end{array}$ & 6 & 14.9 & 14.9 & 97.0 \\
Total & 202 & 100.0 & 100.0 & 100.0 \\
\hline
\end{tabular}

Spielberger's State-Trait Anxiety Inventory (STAI)

The STAI is an instrument for measuring anxiety levels via self-report. It contains two distinct subscales: the State Anxiety Scale (SAnxiety) evaluates the respondents' current state of anxiety by asking how they feel at the moment to measure subjective feelings of apprehension, tension, nervousness, worry, and

activation/arousal of the autonomic nervous system. The Trait Anxiety Scale (T-Anxiety) 
evaluates the more general and long-standing qualities of trait anxiety by asking the respondents how they generally feel to measure "anxiety proneness," including general states of calmness, confidence, and security.
All items for each subtest are rated on a 4point scale. Scores range from 20 to 80 ; higher scores indicate greater anxiety. A cut point of 39-40 has been suggested to detect clinically significant symptoms for the S-Anxiety Scale (12).

Table 2. The results of the anxiety test STAI

\begin{tabular}{|c|c|c|c|c|c|}
\hline & & Frequency & Percent & Valid Percent & $\begin{array}{l}\text { Cumulative } \\
\text { Percent }\end{array}$ \\
\hline \multirow[t]{4}{*}{ Valid } & $\begin{array}{l}\text { High scores on S-Anxiety } \\
\text { Scale (1) }\end{array}$ & 12 & 5.9 & 5.9 & \\
\hline & $\begin{array}{l}\text { High scores on T-Anxiety } \\
\text { Scale (2) }\end{array}$ & 14 & 6.9 & 6.9 & 12.9 \\
\hline & 1 and 2 & 176 & 87.1 & 87.1 & 100.0 \\
\hline & Total & 202 & 100.0 & 100.0 & \\
\hline
\end{tabular}

Evaluation of blood pressure control

Most of the patients reported high blood pressure occurring especially at night. During each evaluation, BP was measured twice by trained Medical Students at the participant's home or at one of the clinical research facilities. $94.6 \%$ of the patients had longstanding hypertension; $78.22 \%$ were regularly taking antihypertensive drugs according to the ESH/ESC Guidelines for the management of arterial hypertension; $21.78 \%$ were on monotherapy, $72.28 \%$ were on combined drug therapy. Blood pressure elevation was observed despite regular administration of antihypertensive treatment and was associated by anamnesis with the onset of symptoms of increased anxiety and depression. 91 patients (45\%) had been diagnosed with concomitant disease(s) of the cardiovascular system and the circulatory organs (according to the Tenth Revision of the International Statistical Classification of Diseases and Related Health Problems).

Stress stimulates the release of various hormones, which can result in elevated blood glucose levels (13). $11.4 \%$ of patients in the studied group were diagnosed with diabetus mellitus; of them $9.91 \%$ were taking medications and $1.49 \%$ were taking insulin.

Family history

Our research showed that $36.14 \%$ of the studied group had lineal relatives or collateral relatives of the second degree suffering from a mental and/or cardiovascular disease (Figure 1).

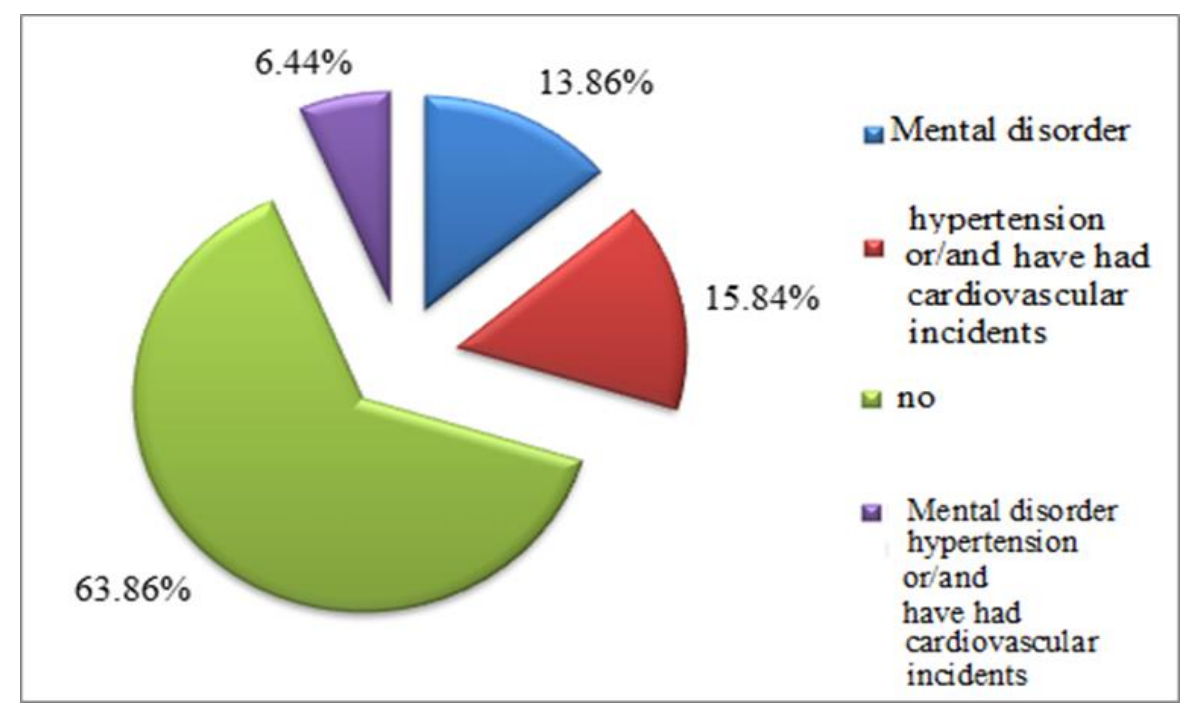

Figure 1. Do you have any blood relatives with: 


\section{RESULTS AND DISCUSSION}

Most of the patients who sought a psychiatrist's help due to increased anxiety and depression in combination with impaired blood pressure control were women $(72.3 \%)$. The

mean $( \pm \mathrm{SD}$ ) age was $60.1 \pm 15.0$ years (range $24-85$ years) for the men and $66.3 \pm 10.6$ years (range $23-86$ years) for the women.
The mean $( \pm \mathrm{SD})$ systole pressure of the patients was $146.3 \pm 14.2 \mathrm{mmHg}$ (range $110-$ $210 \mathrm{mmHg}$ ) and their mean diastole pressure was $88.5 \pm 9.9 \mathrm{mmHg}$ (range $55-120$ $\mathrm{mmHg}$ ). Those values were based on measurements twice by trained Medical Students. The values reported by anamnesis were higher. (Figure 2)

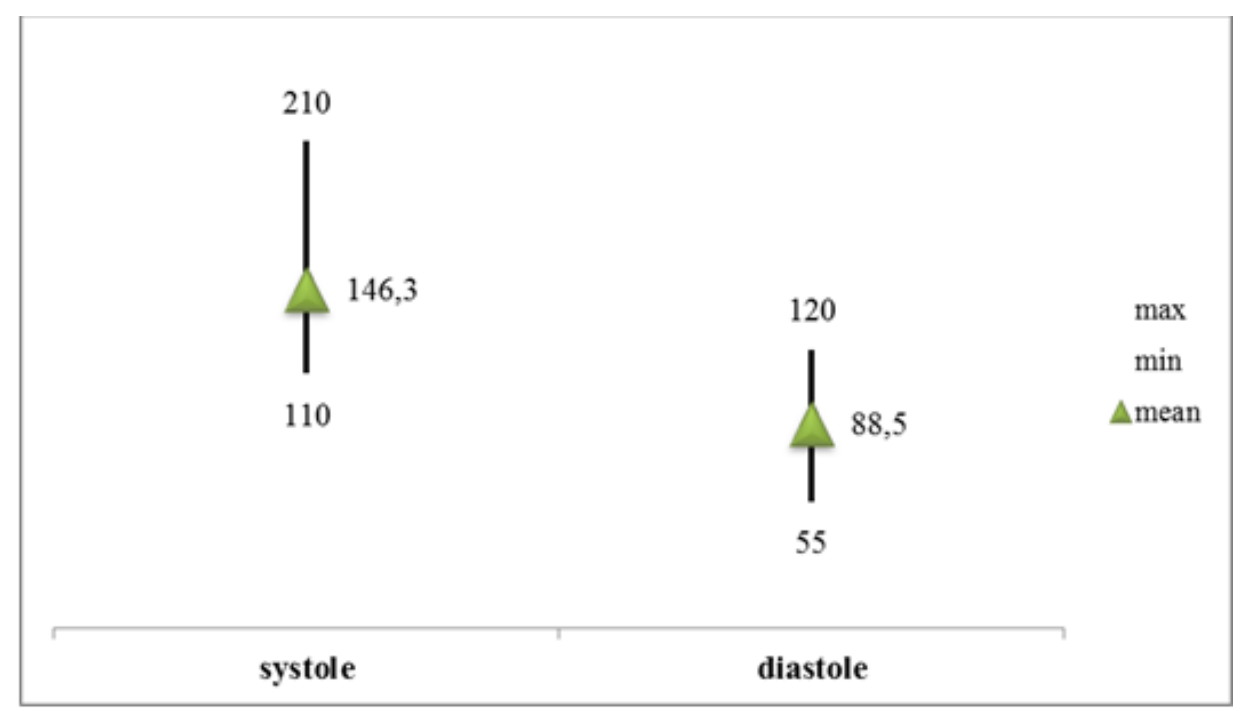

Figure 2. The mean $( \pm \mathrm{SD})$ systole pressure of patients was $146.3 \pm 14.2 \mathrm{mmHg}$ (range $110-210$ $\mathrm{mmHg}$ ), and diastole pressure was $88.5 \pm 9.9 \mathrm{mmHg}$ (range $55-120 \mathrm{mmHg}$ ). (before anxiolytic treatment)

During a guided interview, the patients reported somatovegetative symptoms: insomnia or sleep disorders (trouble falling asleep, early morning awakening, sleep inversion) was observed in $83.2 \% \quad(168$ patients); changes in appetite and/or body weight - in $74.3 \%$ (150 patients); decreased libido - in $48.5 \%$ (98 patients); headache - in
$77.7 \%$ (157 patients); tachycardia - in $69.3 \%$ (140 patients).

According to the diagnosis and the evaluation of the severity of the condition, anxiolytic therapy with 4 main groups of drugs with different mechanism of action, administered alone or in combination, was prescribed by a psychiatrist. (Table 3)

Table 3. Psychiatrist-prescribed therapy - individual administration and combinations

\begin{tabular}{|ll|l|l|l|l|}
\hline & Frequency & Percent & Valid Percent & Cumulative \\
Percent
\end{tabular}

1. neuroleptics 2. antidepressants 3. Deanxit 4. benzodiazepines 
All patients underwent 1-month, 3-month and 6-month follow-up examinations. At the 6month follow-up, $97 \%$ of the hypertensive patients reported optimization of their blood pressure and reduction of the somatovegetative symptoms. At the repeated STAI test, all patients showed lower levels on the S-Anxiety scale.

\section{CONCLUSION}

Our results indicate that in patients with an anxiety disorder accompanied by hypertension, the inclusion of an anxiolytic drug in addition to the antihypertensive therapy leads to stabilization of the blood pressure, reduction of somatovegetative symptoms and decrease in the anxiety levels.

\section{ACKNOWLEDGEMENT}

This work was supported by Grant no. 10/2014 from the Fund for Scientific and Mobile project from Faculty of Medicine at the Trakia University, Stara Zagora, Bulgaria.

\section{ABBREVIATIONS}

BP- blood pressure

NTS- nucleus of the solitary tract

RAS- renin-angiotensin system

STAI -Spielberger's State-Trait Anxiety Inventory

\section{REFERENCES}

1. Gasperin, D., Netuveli, G., Dias-da-Costa, J.S., Pattussi ,M.P., Effect of psychological stress on blood pressure increase: a metaanalysis of cohort studies. Cad Saude Publica, 25(4):715-26,2009.

2. Lucini, D., Di Fede,G., Parati, G., Pagani,M., Impact of chronic psychosocial stress on autonomic cardiovascular regulation in otherwise healthy subjects. Hypertension, 46(5):12016, 2005.

3. Groeschel, M., Braam, B., Connecting chronic and recurrent stress to vascular dysfunction: no relaxed role for the reninangiotensin system. Am J Physiol Renal Physiol. 300(1):F1-10, 2011.

4. Harrison, D.G., Guzik ,T.J., Lob, H.E., Madhur, M., Marvar, P.J., Thabet, S., Vinh, A. and Weyand, C., Inflammation, Immunity and Hypertension. Hypertension, 57(2): 132-140, 2011.
5. Marvar, P., Vinh, A., Thabet,S., Lob, H.E., Geem, D., Ressler, K. and Harrison D.G., T lymphocytes and Vascular Inflammation Contribute to Stress-Dependent Hypertension. Biol Psychiatry, 71(9): 774-782, 2012.

6. Daubert, D.L.,McCowan, M., Erdos, B. and Scheuer, D., Nucleus of the solitary tract catecholaminergic neurons modulate the cardiovascular response to psychological stress in rats. $J$ Physiol., 590(Pt 19): 4881-4895, 2012.

7. Bremmer, M.A., Deeg,D.J., Beekman, A.T., Penninx,B.W., Lips,P., Hoogendijk, W.J., Major depression in late life is associated with both hypo- and hypercortisolemia. Biol Psychiatry, 62(5):479-86, 2007.

8. Penninx, B.W., Beekman,A.T., Bandinelli, S., Corsi ,A.M., Bremmer ,M., Hoogendijk, W.J., Guralnik J.M., Ferrucci, L., Late-life depressive symptoms are associated with both hyperactivity and hypoactivity of the hypothalamo-pituitary-adrenal axis. Am J Geriatr Psychiatr ., 15(6):522-9,2007.

9. Manenschijn, L., Schaap, L., van Schoor, N.M., van der Pas ,S., Peeters, G.M., Lips ,P., Koper J.W., van, E.F., High longterm cortisol levels, measured in scalp hair, are associated with a history of cardiovascular disease.; J Clin Endocrinol Metab.,98(5):2078-83, 2013.

10.Nabi, H., Chastang, J.F., Lefèvre, T., Dugravot, A., Melchior, M., Marmot, M.G., Shipley, M.J., Kivimäki, M., SinghManoux,A., rajectories of depressive episodes and hypertension over 24 years: the Whitehall II prospective cohort study Hypertension., 57(4):710-6, 2011.

11.Matthews, K.A., Katholi, C.R., McCreath, H., Whooley,M.A., Williams,D.R., Zhu,S., Markovitz, J.H., Blood pressure reactivity to psychological stress predicts hypertension in the CARDIA study. Circulation, 110(1):74-8, 2004.

12.Julian,L., Measures of Anxiety. Arthritis Care Res (Hoboken), 63 Suppl 11:467-72, 2011.

13.Surwit, R.S., Schneider, M.S., Feinglos, M.N, Stress and Diabetes Mellitus.Diabetes Care, 15(10):1413-22, 1992. 